\title{
ЧЕЛОВЕК И ВСЕЛЕННАЯ - ПРОБЛЕМА ВЗАИМОСВЯЗИ
}

Тарароев Яков Владимирович

Введение.

Вопросами взаимосвязи себя самого и окружающего его мира, человек стал задаваться ещё на самых ранних этапах становления своего самосознания. “Что есть человек?”, “Каково его место в мире?”, “Каково его предназначение и что ожидает его в будущем?” - эти и целый ряд подобных вопросов всегда волновали его и были для него актуальны. Ещё в античности стало понятно, что наиболее полный ответ на подобные вопросы можно получить, имея достаточно широкое представление о том, что представляет собой окружающий мир. В частности, Платон в диалогах “Государство” и “Тимей” показывает, что вопрос “о природе человека (душа, познавательные силы души, отношение души и тела, вселение души в тело и её судьба после смерти человека) ${ }^{1}$, требует для своего решения именно самых общих представлений о мире, которые он и описывает в заключительной 10-ой книге "Государства" и диалоге “Тимй”, Тот же самый подход в познании человеческой природы наблюдался и позднее. Как пример, можно указать Джордано Бруно с его работами “О причине, начале и едином" и “О бесконечности, вселенной и мирах"з. Однако, несмотря на подход, используемый Платоном, Дж. Бруно и некоторыми другими философами, в целом взаимосвязь Человека и Вселенной носила скорее метафизический, а не натуралистический характер, оставаясь связью на уровне аналогий, параллелей и образов.

Существенным образом ситуация в этом вопросе стала меняться со второй половины ХХ столетия, когда естественные науки в своём развитии дошли до определённого уровня и смогли зафиксировать, прежде всего физические 4 связи между Человеком и Вселенной, и даже выразить их количественно. Фиксация совокупности этих связей в научной литературе получило название антропного принципа.

В самом общем виде суть антропного принципа заключается в том, что если рассматривать человека и человеческое сообщество как сложную, высокоорганизованную систему, то необходимо констатировать, что для существования такой системы необходим целый комплекс особых, специфических условий, которые касаются всех уровней организации материи: физического, химического, биологического и социального. Иными словами, сложные, высокоорганизованные системы, к которым относятся человек и человеческое сообщество, могут возникнуть и развиваться при некоторых, строго определённых внешних условиях, причём спектр вариации этих условий весьма ограничен. Впервые на это обстоятельство обратил внимание

\footnotetext{
${ }^{1}$ Платон. Собрание соч. : В 4-х т.: Пер. с древнегреч. - М., Мыслль. - ТЗ: 1994. - 657 с., С.530.

${ }^{2}$ Как отмечает сам Платон: ”Мы решили, что, коль скоро Тимей являет собой среди нас самого глубокого знатока астрономии, и главнейшим своим занятием сделал познание природы всех вещей, он и будет говорить первым, начав с возникновения космоса и закончив природой человека“" - Тимей, 27-b.

${ }^{3}$ Бруно Дж. Изгнание торжествующего зверя. О причине, начале и едином. - Минск: Харвест, 1999. - 480 с., Бруно Дж. О Бесконечности, вселенной и мирах. - М.: Сочэкгиз, 1936 - 245 с.

4 Наиболее уместным здесь было бы изначальное понимание термина “физические”, происшедшего от древнегреческого “фи́бเৎ” - природа.
} 
советский учёный Г.М. Идлис ещё в 1958 году ${ }^{5}$ Позднее эту тему развил, сформулировав сильный и слабый антропный принцип и дав ему естественнонаучное объяснение, британский астрофизик Картер ${ }^{6}$ В 80-х годах XX столетия эта тематика “набирала обороты”; выходили уже целые монографии. Классической считается работа Дж. Д. Барова и Ф.Дж. Типлера ${ }^{7}$. С последующим развитием современных научных знаний интерес к этой тематике не ослабевал. В последнее десятилетие XX столетия и в первые годы нового столетия продолжали выходить работы, в которых обращалось внимание на новые аспекты антропного принципа и его новые следствия и интерпретации. В этом же ряду стоит недавно вышедшая монография известного физика и космолога М. Риса ${ }^{8}$.

Однако, несмотря на активную работу научного сообщества, эта тема ещё очень далека до исчерпания. Стремительное развитие современной науки вскрывает всё новые и новые объекты, виды и типы связей в природе, которые по прошествии совсем небольшого промежутка времени осознаются как ключевые связи при рассмотрении проблемы соотношения Человека и Вселенной. Конечно, эти связи, как правило, опосредованы, поскольку сам Человек не является непосредственно космическим объектом, а выступает скорее элементом других, относительно автономных систем: прежде всего биологического уровня организации материи и геофизической системы. Очевидность этого положения стала ясной ещё с первой половины XX столетия. Современное развитие естествознания всё более “урезает” “автономизацию” этих систем, вскрывая всё новые факты зависимости их как от глобальных, фундаментальных свойств материи, так и от пространственно-локальных (по космологическим меркам) условий их существования. Эти обстоятельства делают обоснованной попытку последовательно систематизировать взаимосвязь основных факторов, определяющих возникновение, существование и развитие этих систем, и, следовательно, и Человека как их составной части. Анализ и систематизация локальных (в пределах Солнечной системы и Земли) факторов, обуславливающих возникновение и развитие биологического и социального уровней организации материи, в силу их многоплановости, требует рассмотрения в отдельной работе. В данной работе рассматриваются только основные глобальные свойства материи, необходимые для существования её физического и химического уровня организации.

1. Связи Человека и Вселенной: физический ${ }^{9}$ аспект.

\footnotetext{
5 Идлис Г.М. Основные черты наблюдаемой астрономической Вселенной как характерные свойства обитаемой космической системь. // Изв. Астроф. Ин-та АН КазССР. - 1958. - Т.7 - С.39-54.,

${ }^{6}$ Картер Б. Совпадения больших чисел и антропологический принцип в космологии. // Космология: теория и наблюдения. - М.: Мир. - 1978. - С. 369-379.,

${ }^{7}$ Barrow J.D., Tipler F.J. The Anthropic Cosmological Principle. - Oxford: Oxford Univ. Press., 1986. - 388 p.

${ }^{8}$ Рис М. Наша космическая обитель. Пер с англ. - Москва-Ижевск: Институт компьютерных исследований, 2002 $192 \mathrm{cmp}$.

9 В данном контексте “физический” уже подразумевает современное понимание этого термина как совокупности знаний о наиболее фундаментальных свойствах материи.
} 
Физический уровень организации материи в значительной степени определяющий все остальные уровни. В контексте рассматриваемого вопроса можно даже утверждать, что во взаимосвязи Человек - Вселенная, этот уровень играет ключевую роль, хотя, конечно, данная взаимосвязь не ограничивается только им. Сам Человек и биологический уровень организации материи, к которому он принадлежит и окружающий его мир в целом, есть, кроме всего прочего, физическая система, подчиняющаяся определённым физическим закономерностям. Кроме того, этим же закономерностям подчиняются и связи между Человеком и окружающим его миром.

В рамках поставленной задачи физический уровень организации материи несколько условно можно представит в виде двух форм - вещества и поля. Условность такого представления объясняется целым рядом обстоятельств, к которым можно отнести современные достижения в области теории суперсимметрии, ставящей себе задачу выработать единый подход к описанию вещества и поля ${ }^{10}$, а так же открытое в 1997 году ускоренное расширение Вселенной и, связанная с этим, проблема причин этого ускорения, называемая “тёмная энергия”"11.

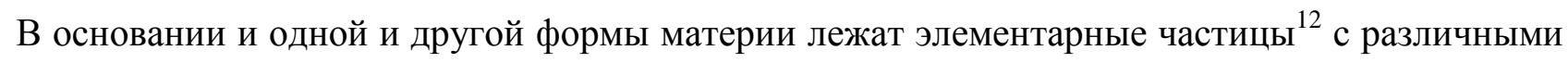
свойствами. Если говорить языком физики, то разница между одними и другими заключаются в том, что частицы вещества подчинены принципу Паули, а частицы поля нет ${ }^{13}$. Их различие можно попытаться выразить и более нестрогим языком, в обыденном представлении, которое исключает целый спектр специфических физических представлений: в одной точке пространства может находиться только одна частица вещества, но несколько частиц поля ${ }^{14}$. В силу этого и других различий, способностью составлять сложные высокоорганизованные системы обладают только частицы вещества, а частицы поля выступают лишь в качестве взаимодействия, т.е. связей, как между элементами системы, так и между системами. И определённые свойства поля, и определённые свойства вещества является необходимыми для существования человека как сложной высокоорганизованной системы.

\section{1. Связь полевой формы материи и Человека.}

Как было сказано выше, физические поля “обеспечивают” взаимодействие и связь между различными элементами физических систем. Эту функцию для достаточно стабильных ${ }^{15}$ сложных систем выполняют четыре вида взаимодействий: слабое, сильное, электромагнитное,

${ }^{10}$ Подробнее о суперсимметрии см. например: Вайнберг С. Квантовая теория полей. Том 3. Суперсимметрия. Перевод с англ. - М.: ФАЗИС, 2002. - 458 с.

11 Подробнее об ускоренном расширении Вселенной и “тёмной энергии” см. ниже.

12 Следует помнить, что эти частицы не являются частицами в обыденном понимании, поскольку им присущ т.н. “корпускулярно-волновой дуализм”, т.е. они способны, в различных ситуациях проявлять свойства частицы и волны.

13 Частицы, подчиняющиеся принципу Паули, называют фермионами, не подчиняющиеся - бозонами.

14 С точки зрения физики, строго формально, это утверждение не вполне правомочно хотя бы потому, что элементарные частицы не могут быть локализованы в точке пространства, однако для наглядности различий между веществом и полем оно может быть использовано.

15 Здесь подразумевается то, что кроме вышеназванных полей существуют и другие. В частности, физический вакуум описывается скалярным полем ф и некоторыми другими, однако в вакууме существуют только виртуальные (короткоживущие) частицы вещества, поэтому его считают только потенциальнылм многообразием. Актуально физический вакуум представляет собой пустоту. Это относится и к низкоэнергетическому физическому вакууму, который окружает нас всюду, и к высокоэнергетическому, из которого появилась наша Вселенная. 
гравитационное. В контексте рассматриваемого вопроса особый интерес представляют последние три.

А. Сильное или внутриядерное взаимодействие, ответственно за взаимодействие протона и нейтрона внутри атомного ядра, а также за термоядерные реакции, при которых происходит синтез тяжёлых ${ }^{16}$ элементов. Очевидна первостепенная значимость этого вида взаимодействия. Атомные ядра являются основой тех “первокирпичиков”, из которых состоят все стабильно существующие сложные высокоорганизованные системы. Можно с уверенностью утверждать, что основу такого стабильного существования закладывает именно определённый баланс сильного взаимодействия. Кроме того, этот же баланс определяет и протекание термоядерных реакций, без которых говорить о многообразии химических элементов просто не представлялось бы возможным.

Б. Электромагнитное взаимодействие - взаимодействие электростатических и магнитных зарядов. Характеризуется несколькими постоянными, основной из которых является скорость электромагнитной волны (света) в вакууме $c$, как максимальная скорость передачи любого сигнала (информации). Оно лежит в основе почти всех процессов и явлений вокруг нас - физических, химических и биологических. В частности, именно электромагнитное взаимодействие обеспечивает взаимосвязь клеток организма, создаёт “защитный пояс" Земли от космических частиц и лучей высоких энергий. Кроме того, это взаимодействие играет ключевую роль в “завершении” строительства атомов. Именно электромагнитное взаимодействие ответственно за взаимодействие между электронами и протонами в атоме. И именно определённый баланс этого взаимодействия с сильным взаимодействием позволяет стабильно существовать сложной системе: нуклоны (протоны + нейтроны) + электрон.

В. Гравитационное взаимодействие. Самое универсальное взаимодействие, т.к. в нём участвуют все частицы с ненулевой массой покоя, т.е. все частицы вещества. Характеризуется постоянной тяготения. Однако, в силу своего “слабодействия” ощутимо только на астрономических и космологических масштабах, поэтому оно играет ключевую роль во всех подобных процессах, в том числе и в тех, от которых существенным образом зависит существование биологического уровня организации материи, и его составной части - Человека. Рассмотрим основные процессы. Во первых, гравитационное взаимодействие ответственно за существование всех астрономических систем, в том числе и Солнечной. Именно баланс гравитационных сил обеспечивает стабильное движение планет (в том числе и Земли) вокруг Солнца по замкнутым орбитам. Этот фактор является одним из самых основных для возникновения жизни, поскольку феномен жизни возможен только при наличии процесса

\footnotetext{
${ }^{16}$ Иметься в виду синтез всех элементов, тяжелее водорода и гелия.
} 
метаболизма - “прокачки” энергии через открытую систему ${ }^{17}$. Жизнь на Земле существует порядка 3,8 миллиардов лет, и всё это время её существование и развитие обеспечивалось потоком солнечной энергии. В случае отсутствия замкнутых орбит, никаких сложных форм организации материи сложнее химической в природе не могло бы существовать. Во вторых, стабильность Солнечной системы зависит от стабильности её системообразующего элемента - Солнца. Стабильное существование Солнца определяется балансом сил гравитационного взаимодействия и давления плазмы внутри его. В случае если бы этот баланс не был таков, каковым он является в нашей Вселенной, то Солнце “выгорело” бы слишком быстро или, наоборот, не “зажглось” бы вообще. Кроме того, гравитационное взаимодействие является основным в общей динамике эволюции Вселенной. Если бы оно было больше или меньше наблюдаемого, то Вселенная в первом случае слишком быстро закончила бы эволюцию в целом, и для возникновения сложных высокоорганизованных (биологических) форм материи у неё бы не осталось времени ${ }^{18}$, а во втором, при медленной эволюции, во Вселенной не смогли бы образоваться ни галактики, ни звёзды. В контексте проблемы образования галактик существенным является ещё один фактор, а именно длина волны первичных возмущений плотности тех специфических состояний материи, которые были характерны для ранней Вселенной. Для образования галактик, звёздных и планетарных систем необходим определённый баланс между гравитационным взаимодействием и первичными возмущениями плотности. В случае если бы при том же гравитационном взаимодействии первичные возмущения были бы значительно меньше, то галактики, возможно, никогда не сформировались, и возникновение звёзд и планет было бы маловероятным. Наоборот, более сильные возмущения плотности вели бы к формированию супермассивных чёрных дыр. Наблюдаемые первичные возмущения плотности являются оптимальными для существования устойчивых астрономических систем.

Д. Размерность пространства-времени ${ }^{19}$. Этот фактор был вынесен отдельным подпунктом, поскольку он является существенным для двух последних взаимодействий - электромагнитного и гравитационного. Было установлено, что только в трёхмерном пространстве возможен требуемый баланс. В пространстве большего числа измерений орбиты электронов вокруг ядра и планет вокруг Солнца будут не замкнуты и, следовательно, эти системы, как целое, не смогут стабильно существовать. А в их отсутствие невозможно говорить о существовании химических и

\footnotetext{
17. См. например Моисеев Н.Н. Человек и биосфера: опыт системного анализа и эксперименты с моделями - М.: Наука. - 1985 - 271 с., Моисеев Н.Н. Идеи естествознания и общественные науки - М.: ВЦ АН СССР - 1991 . - 55 с.

18 Солнце является звездой второго поколения, которое сформировалась из остатков уже “сгоревшей” звезды, (или нескольких звёзд) вследствие чего первичное протосолнечное облако было обогащено тяжёлыми химическими элементами, что явилось необходимым условием для возникновения жизни.

${ }^{19}$ На самом деле в этом подпункте речь будет идти только о размерности пространства, поскольку предположение о размерности времени $\mathrm{N}>1$ несколько экзотично, хотя такое предположение формально вполне допустимо. Представляет определённый интерес, насколько такая размерность времени будет сказываться на стабильном существовании сложноорганизованных систем.
} 
биологических систем вообще, и Человека в частности. В пространстве меньшего числа измерений сложные системы не смогли бы существовать по определению.

Е. “Тёмная энергия”. Если бы эта работа писалась до 1997 года, здесь можно было бы закончить анализ влияния свойств полевой формы материи на существование и развитие, прежде всего, биологических систем, в силу исчерпания предмета анализа. Однако в 1997 году было сделано открытие эпохальной значимости, интерпретация которого будет способна существенным образом изменить наши взгляды на саму материю, на её основания, и на окружающий мир в целом. Речь идёт об ускоренном расширении Вселенной и вызывающей его “тёмной энергии”. Это ускорение было обнаружено при наблюдении очень удалённых сверхновых звёзд. До этого времени считалось, что Вселенная расширяется ${ }^{20}$ с замедлением по степенному ${ }^{21}$ закону a(t) $\mathrm{t}^{2 / 3}$. Однако, сделанное открытие говорило о том, что закон расширения другой. Сразу же возник вопрос о причинах этого расширения. Оставаясь (для наглядности) в рамках ньютоновской механики, можно утверждать, что там, где есть ускорение, должна быть и сила, а действию силы соответствует энергия. Этот же вывод справедлив и в рамках общей теории относительности (ОТО) Эйнштейна. Энергию - причину ускоренного расширения Вселенной назвали “тёмной”, поскольку никаким другим образом, кроме ускоренного расширения Вселенной, она себя не проявляет. Эта энергия обладает примечательными свойствами и представляет собой антигравитационное явление, поскольку, в отличие от гравитации она не притягивает, а отталкивает тела, имеющие массу. По своей “мощности” она превосходит гравитационное взаимодействие, хотя действует даже не на астрономических, а на космологических расстояниях. Первым предположением о природе тёмной энергии была гипотеза о том, что ускоренное расширение Вселенной вызывает физический вакуум. Согласно современной инфляционной парадигме, одним из создателей которой является А.Д. Линде, Вселенная, возникнув из высокоэнергетического физического вакуума, на самых ранних этапах своей эволюции расширялась по экспоненциальному ${ }^{22}$ закону $\mathrm{a}(\mathrm{t}) \sim \mathrm{e}^{t}$. Нетрудно заметить ${ }^{23}$, что это расширение $\mathrm{c}$ ускорением. Согласно различным сценариям, за время $\mathrm{t} \sim 10^{-35}$ секунды её “размеры” увеличились с $10^{-33}$ до $10^{10^{4}}$ см. и может быть намного более 24 . Именно специфика высокоэнергетического физического вакуума порождает такие темпы расширения и вполне оправданно, что наблюдаемое ускоренное расширение Вселенной пытались объяснить действием физического вакуума. Однако

\footnotetext{
${ }^{20}$ Когда речь идёт о расширении Вселенной, необходимо понимать, что это расширение представляет собой не просто удаление одних объектов от других. Это удаление является только следствием расширения самого пространства, которое заключается в увеличении расстояния между любыми двумя его точками. Физической величиной, количественно характеризующей это расширение, является масштабный фактор a(t).

${ }^{21}$ Как нетрудно заметить, ускорение (т.е. вторая производная) по этому закону есть величина отрицательная.

22 По этому же закону происходит процесс финансовой инфляции и все остальные процессы, где скорость изменения какого либо параметра (в случае с финансовой инфляцией это денежная масса) прямо пропорциональна самой изменяемой величине. Именно поэтому данная стадия называется инфляционной.

${ }^{23}$ Взяв вторую производную.

${ }^{24}$ Подробнее о самой инфляционной космологии и проблемах, которые она решает и ставит см. Линде А.Д. Физика элементарных частии и инфляционная космология. - М.: Наука, 1990. - 256с.
} 
дальнейшие наблюдения поставили под сомнения такое объяснение, поскольку наблюдаемые параметры расширения не совсем согласовывались с теоретически предполагаемыми параметрами при условии, что причиной расширения является физический вакуум. В настоящее время, повидимому, можно предположить, что “тёмная энергия” есть некая “многокомпонентная субстанция". Кроме физического вакуума её компонентой могут быть уже известные формы материи - квинтэссенция ${ }^{25}$. Выдвигаются предположения и о том, что таковой может быть ещё неизвестная форма материи - фантомная энергия ${ }^{26}$. Достоверно определить природу тёмной энергии пока не представляется возможным. Однако, несмотря на это, постоянная, определяющая это взаимодействие, уже достаточно точно определена. Это космологическая ${ }^{27}$ постоянная $\Lambda$. Её величина составляет $\Lambda \sim 10^{-56} \mathrm{~cm}^{-2}$. В настоящую эпоху именно определённое соотношение между притягивающим гравитационным и отталкивающим космологическим взаимодействиями обеспечивает ту динамику эволюции Вселенной, которую мы наблюдаем. Тот факт, что во Вселенной существуют биологические и социальные системы говорит о том, что этот баланс в пришлом и настоящем благоприятен для их существования, хотя совсем нетрудно представить себе картину, когда это было бы не так. Относительно будущего, в силу неопределённости природы “темной энергии”, однозначного ответа нет. Некоторые из аспектов влияния ускоренного расширения на существования сложных материальных систем рассмотрены автором в другой работе ${ }^{28}$.

\section{2. Связь вещественной формы материи и Человека.}

В окружающем нас мире существуют значительное множество частиц вещества, отличающихся друг от друга своими характеристиками. Каждая частица участвует в одном или нескольких видах взаимодействий, поэтому их характеристиками являются различные параметры. Так масса характеризует степень участия частицы в гравитационном взаимодействии, электрический заряд - в электромагнитном, цветовые заряды - в сильном взаимодействии, и “заряд” слабого взаимодействия - в слабом. Рассмотрим лишь наиболее важные. К ним, безусловно, относятся частицы, составляющие атомное ядро и атом в целом.

Как уже говорилось выше, основными взаимодействиями, ответственными за существование атомных ядер и соответствующих им атомов являются соответственно сильное и

\footnotetext{
${ }^{25}$ Гинзбург. В.Л. О некоторых успехах физики и астрономии за последние три года. // Успехи физических наук - 2002. - Том 172, №2. - C.213-219.

${ }^{26}$ См. например Vinod B. Johri Phantom Cosmologies // http://lanl.arxiv.org/abs/astro-ph/031129 v3

27 В литературе её иногда ещё называют $\Lambda$ - член. Это связанно с тем, что впервые “силы” космологического отталкивания ввёл в рассмотрение А. Эйнштейн в виде $\Lambda$ - члена в своё тензорное уравнение. Такой подход Эйнштейна был обусловлен его неприятием идеи метрической нестационарности Вселенной, которая следовала из решения его уравнения при учёте того, что гравитация является единственным “глобальным” взаимодействием во Вселенной. Для компенсации действия гравитации, и для сохранения, тем самым стационарного состояния Вселенной, в 1917 году, сразу после создания ОТО он предложил гипотетические “силы отталкивания”. Однако позднее, после вновь полученного нестационарного решения А.А. Фридмана, он отказался от этой идеи. Подробнее см. Пайс А. Научная деятельность и жизнь Альберта Эйнштейна. Пер. с англ. - М.: Наука, 1989 - 558 с.

${ }^{28}$ Тарароев Я.В., Христианская эсхатология в контексте современной космологии // Замысел Бога в теориях космологии, - Издательство Санкт-Петербургского университета, 2004,- С.177- 184.
} 
электромагнитное взаимодействия. Существование атомного ядра обеспечивает взаимодействие кварков, из которых состоят протоны и нейтроны, и которые имеют цветовой заряд. Существование атома обеспечивается электромагнитным взаимодействием между электроном и протоном. Соответственно, все эти взаимодействия находятся в некотором равновесии, что предполагает сбалансированность цветовых и электрических зарядов кварков и электрического заряда электрона. В случае если бы соответствующие заряды этих частиц были бы другими (по величине), это привело бы к нарушению этого баланса и невозможности существования атома, и, следовательно, любых более сложных форм материи. Кроме того, все частицы вещества участвуют в гравитационном взаимодействии и за этот процесс “ответственность несёт” их масса. В случае её изменения баланс гравитационного взаимодействия нарушился бы и, в конечном итоге, это привело бы к тому, что Вселенная стала бы другой, не способной к возникновению и существованию сложных высокоорганизованных систем ${ }^{29}$. Особенно актуальным это положение становится в контексте определения природы “тёмного вещества”. Напомним, что согласно современным взглядам, основной вклад, около 70 \% в плотность Вселенной вносит “тёмная энергия”, порядка $25 \%$ - “тёмное вещество”, и порядка $5 \%$ - видимое вещество ${ }^{30}$. В ряде работ рассмотрены различные “претенденты” на звание “тёмного вещества”. Это, прежде всего, массивные гипотетические слабовзаимодействующие частицы, которыми, в случае наличия у них большой массы, могут быть и нейтрино, гипотетические маломассивные частицы аксионы, частицы, существование которых следует из теории суперсимметрии, гипотетические сверхтяжелые частицы. Развитие современной физики и космологии вскрывает всё новые и новые связи между миром элементарных частиц и условиями, необходимыми для существования сложных материальных систем, в том числе и Человека. Как пример, можно указать недавнюю работу $^{32}$, где в контексте антропного принципа, речь идёт о массе нейтрино и её влияния на образование и эволюцию галактик, без чего невозможно было бы образование звёзд и планет. Этот и многие другие аспекты данной проблемы требуют дальнейшего исследования.

\section{2. Связи Человека и Вселенной: химический аспект ${ }^{33}$.}

\footnotetext{
${ }^{29}$ Так, например, если бы масса электрона (при тех же массах протона и нейтрона) была бы в два раза больше, то протекание протон-протонного цикла в недрах звёзд типа Солнца был бы невозможным, поскольку эта реакция возможна только тогда, когда энергия покоя электрона меньше разницы между энергии связи протона и нейтрона в ядре дейтерия и разницы в энергиях покоя протона и нейтрона, которая, в свою очередь, ограничена возможностью протекания термоядерного синтеза тяжёлых элементов.

${ }^{30}$ Гинзбург. В.Л. О некоторых успехах физики и астрономии за последние три года. // Успехи физических наук - 2002. - Том 172, №2. - C.213-219.

${ }^{31}$ Albrecht Andreas, Frieman Joshua A., Trodden Mark. Early Universe Cosmology and Test of Fundamental Physics: Report of the P. 4.8 Working Subgroup, Snowmass 2001 // http://lanl.arxiv.org/abs/hep-ph/011108 v1, Kamionkowski Marc. New Views of Cosmology and the Microworld. // http://lanl.arxiv.org/abs/hep-ph/0210370v1, Ellis By John. Dark Matter and Dark Energy: Summary and Future Directions // http://lanl.arxiv.org/abs/ astro-ph/0304183v1

${ }^{32}$ Max Tegmark and Alexander Vilenkin Anthropic predictions for neutrino masses. // http://lanl.arxiv.org/abs astroph/0304536v1

33 Данное название несколько условно. Правильнее было бы назвать “физические основания химического аспекта”, поскольку речь в нём будет идти главным образом о физических условиях, необходимых для существования химического уровня организации материи.
} 
Если рассматривать жизнь в целом и Человека, как её составную часть, как сложную, высокоорганизованную систему, то необходимо признать, что химический уровень организации материи в этом рассмотрении играет не менее важную роль, чем физический уровень организации. Человек есть не только физическая, но и химическая система, состоящая из множества химических элементов и соединений. С точки зрения химии, для человеческого существования принципиальными являются несколько факторов:

А. наличие многообразия химических элементов;

Б. наличие условий для возникновения и существования сложных химических соединений;

В. распространенность химических элементов, особо необходимых для существования жизни и Человека.

Конечно, кроме приведенных факторов, возможны и целый ряд других, значимость которых не столь очевидна сейчас, однако может быть оценена в будущем, но в настоящее время эти факторы наиболее очевидные. Рассмотрим их подробнее.

А. Наличие многообразия химических элементов. Этот фактор является ключевым и для существования жизни вообще, и Человека в частности. С точки зрения химии всякое живое, а Человек тем более, есть, прежде всего, система сложным образом взаимодействующих химических элементов. Всего в природе встречаются 83 химических элемента. Из них необходимы для нормального функционирования человеческого организма почти все, за исключением инертных газов, и некоторых других. Можно смело утверждать, что будь химических элементов хотя бы в 2 раза меньше, существование такого феномена, как Человек, было бы под большим вопросом. Наличие такого разнообразия химических элементов определяется двумя факторами: 1. Потенциальной возможностью их существования. Эта возможность обусловлена тремя видами взаимодействий - сильного, электромагнитного и гравитационного. Будь баланс этих взаимодействий иной, и количество химических элементов вообще, и участвующих в “жизненных” процессах в том числе, было бы другое. Так, в частности, если бы сильное взаимодействие было бы менее интенсивное, то его “мощности” не хватило бы, что бы удержать протоны в ядрах тяжёлых элементов. Чем меньше было бы сильное взаимодействие, тем меньше протонов было бы в ядре, и тем легче был бы самый тяжёлый химический элемент, встречающийся в природе. 2. Актуальностью их существования. Актуальное наличие широкого разнообразия химических элементов является одним из основных условий существования сложных высокоорганизованных систем во Вселенной. Между тем, на ранних этапах эволюции во Вселенной было широко распространено всего два химических элемента - водород $(\mathrm{H})$ и гелий $(\mathrm{He})$ и, кроме них, в незначительном количестве присутствовал дейтерий (D) и литий (Li). Всё остальное многообразие появилось позднее, вместе с возникновением звёзд, в которых начали происходить реакции термоядерного синтеза более тяжёлых элементов. Процесс термоядерного синтеза представляет собой усложнение 
относительно простых систем (ядер лёгких элементов), которые включают в себя, в дополнение к уже имеющимся протонам и нейтронам новые, становясь уже ядрами более тяжёлых элементов ${ }^{34}$. В однородной Вселенной этот процесс происходить не мог бы, т.к. для его прохождения необходима концентрация энергии. Местом такой концентрации и становятся звёзды, где “сжатая” гравитационная энергия, через различные физические превращения усложняет ядра лёгких элементов. С этой точки зрения разнообразие химических элементов определяется временем продолжительности существования звезды и температурой в её недрах. Чем дольше живёт звезда, и чем выше температура её ядра, тем больше ядер тяжёлых химических элементов она “производит”. А эти факторы, в свою очередь, определяются гравитационным взаимодействием, причём его “величина” должна лежать в определённых пределах. Если бы оно было больше существующего, то эволюция Вселенной в целом и звёзд в частности происходила бы слишком быстро, и тяжёлые элементы не успевали бы образоваться в таком количестве. С другой стороны, если бы оно было меньше, то его “мощности” не “хватило” для концентрации газа и образования звёзд. Кроме того, в процессе звёздообразования, как уже было сказано выше, существенную роль играют первичные возмущения плотности.

Б. Наличие условий для возникновения и существования сложных химических соединений. Многообразие химических элементов является предпосылкой ещё большего многообразия многообразия химических соединений. Именно это многообразие, с химической точки зрения, предопределяет существование более сложного уровня организации материи - биологического, включая и Человека, в основании которого лежат сложные молекулярные углеродные соединения $^{35}$. Некоторые из них достаточно широко распространены в космосе ${ }^{36}$, другие более редки и уникальны. Однако их наличие возможно только при наличии условий, необходимых для протекания химических реакций взаимодействия элементов. Под условиями здесь, прежде всего, подразумеваются физические условия системы, в которой происходит химическая реакция, такие как температура, объём, давление и др. Можно сказать иначе. Для протекания любой химической реакции необходимо, что бы энергия взаимодействующих частиц была не больше и не меньше определённых величин. Достаточно широкий спектр таких условий, благоприятных для протекания химических реакций образования сложных химических соединений обусловлен двумя факторами: 1. наличием во Вселенной источников энергии, в первую очередь звёзд; 2. общими процессами эволюции Вселенной. Первый фактор значим тем, что наличие во Вселенной источников энергии ${ }^{37}$ создаёт в ней неравновесие физических условий, их своеобразный градиент,

\footnotetext{
${ }^{34}$ Увеличив количество протонов и нейтронов, атомные ядра обладают “запасом” энергии и способны присоединить к себе количество электронов, соответствующе количеству протонов. Сам процесс присоединения (рекомбинации) и соответственно, образования электрически нейтральных атомов осуществляется только тогда, когда атомные ядра каким либо образом покидают звезду и попадают в те физические условия, где температура ниже температуры ионизации.

${ }^{35}$ Подробнее об этом см. например Шкловский И.С. Вселенная. Жизнь. Разум. 6-е. изд., доп. - М.: Наука, 1987. - 320 с.

${ }^{36}$ См. там же.

${ }^{37}$ Кроме звёзд, таковыми являются активные ядра галактик, аккреция вещества на массивные объекты.
} 
области с различными величинами энергии (температуры) где, при наличии соответствующих химических элементов, протекают химические реакции. В то же время наличие областей с низкой энергией (например, газопылевые облака, твёрдые тела и др.) создаёт благоприятные условие для их стабильного существования. Второй фактор так же весьма существенно значим, т.к. определяет среднюю энергию (температуру) во Вселенной в целом. Если бы сложился иной баланс факторов, определяющих метрическую эволюцию Вселенной ${ }^{38}$, и её цикл от расширения до сжатия был бы слишком коротким, то во Вселенной не существовало бы перепадов (градиентов) энергий (температур) и сложные химические соединения не образовывались.

В. Распространенность химических элементов, особо необходимых для существования жизни и Человека. Как уже говорилось выше, значительная часть химических элементов является необходимой для существования жизни. Однако здесь мы рассмотрим только два - углерод (C) и водород $(\mathrm{H})$. Первый лежит в основании всех живых систем, второй является основным компонентом воды, без которой жизнь существовать не смогла бы. Между тем, широкая распространенность углерода так же предполагает определённую “подстройку” на уровне сильного взаимодействия. М. Рис ${ }^{39}$ отмечает, что процесс образования ядра углерода из трёх ядер атома гелия результативен только при условии, что ядро углерода проявляет неожиданное свойство - моду колебаний с очень особой энергией. В противном случае возможность соединения трёх ядер гелия в углерод была бы столь ничтожной, что атомы углерода встречались бы очень редко, и о существовании жизни на его основе не могло бы идти речи. В свою очередь, это свойство ядра углерода зависит от силы сильного взаимодействия. Если бы эта сила была меньше или больше на $1-2 \%$, то этого свойства не существовало. Примерно такая же “подстройка” наблюдается и для водорода. Известный специалист Пол Девис отмечает ${ }^{40}$, что если бы сильное взаимодействие было бы только на 4\% “мощнее”, то при первичном нуклеосинтизе (т.е. образовании ядер лёгких элементов в начальные этапы эволюции Вселенной) первичный “суп” протонов быстро преобразовался бы в дейтерий, который весь синтезировался бы в гелий и водорода в природе просто не существовало бы. Отсутствие водорода явилось бы существенным препятствием для возникновения и функционирования сложных систем, поскольку он является основой для многих элементов таких систем, начиная от звёзд, химических неорганических соединений, в том числе и “универсального растворителя” - воды, и заканчивая сложными органическими соединениями.

\section{Заключение}

Обобщая всё вышесказанное относительно фундаментальных онтологических свойств нашего мира, можно утверждать, что Вселенная, в которой мы живём, в значительной степени

\footnotetext{
${ }^{38}$ Об этих факторах см. выше.

${ }^{39}$ Рис М. Наша космическая обитель. Пер с англ. - Москва-Ижевск: Институт компьютерных исследований, 2002 192 cmp., cmp. 52-53.

${ }^{40}$ P.C.W. Davies Multiverse cosmological models // http://lanl.arxiv.org/abs/astro-ph/0403047, p.7.
} 
“подстроена" под существование сложных, высокоорганизованных, прежде всего биологических систем и их составной части - Человека. “Степень” этой “подстройки” достаточна высока, и позволяет даже некоторым специалистам ${ }^{41}$ говорить о биофиличности нашей Вселенной. Как только был осознан этот факт, сразу же стал вопрос о причинах этой “подстройки”. Здесь возможно два ответа:

1.Вся совокупность этих факторов сложилась случайным образом. Однако это очень маловероятное событие. По утверждению В. Крейга ${ }^{42}$, его вероятность составляет $10^{-123}$.

2.Вся совокупность этих факторов сложилась при целенаправленном воздействии. Это воздействие осуществил Бог, и всё вышесказанное является доказательством существования Бога.

Однако при рассмотрении первого предположения следует учитывать следующий момент. Если возникает одна Вселенная, то вероятность наличия у неё биофиличных свойств равна $10^{-123}$. Однако если возникает $10^{123}$ вселенных, то с вероятностью 1 можно утверждать, что хотя бы в одной из них будут свойства, благоприятные для существования сложных, высокоорганизованных систем. В других вселенных будут другие свойства, возможно не благоприятные для существования Человека. Впервые эту мысль высказал Б.Картер ${ }^{43}$, предложив для объяснения антропного принципа идею “ансамбля миров”. Однако картеровская гипотеза до самого конца XX столетия оставалась спекулятивным предположением, пока советский физик А.Д. Линде не предложил теорию “хаотической инфляции”,44, которая описывала физические механизмы возникновения множественности вселенных. Согласно этой теории, из высокоэнергетического физического вакуума рождается не одна наша, а значительное количество замкнутых ${ }^{45}$ вселенных с различными физическими и геометрическими свойствами. Каждая из вселенных проходит вышеописанную стадию инфляционного расширения, свойства материи пространства и времени внутри неё определяются “местными”, “локальными” условиями высокоэнергетического физического вакуума в момент рождения вселенной. Таким образом, согласно этой теории, мир в целом является существенно неоднородным и представляет собой совокупность своеобразных “пузырей”46 - областей пространства с различными свойствами и состояниями материи, “отгороженных” друг от друга специфическими физическими образованиями - доменными

\footnotetext{
${ }^{41}$ Рис М. Наша космическая обитель. Пер с англ. - Москва-Ижевск: Институт компьютерных исследований, 2002 192 cmp., P.C.W. Davies Multiverse cosmological models // http://lanl.arxiv.org/abs/astro-ph/0403047, P.C.W. Davies How bio-friendly is the universe? // http://lanl.arxiv.org/abs/astro-ph/0403050

${ }^{42}$ Крейг Уильям. Самое начало. (Происхождение Вселенной и существование Бога.) Чикаго: SGP Bох 502, Chicago, IL, 60690-0502 USA, 1987. - $80 c$.

${ }^{43}$ В той самой работе, о которой шла речь в начале: Картер Б. Совпадения больших чисел и антропологический принции в космологии. // Космология: теория и наблюдения. - М.: Мир. - 1978. - С. 369-379.,

${ }_{44}$ См. Линде А.Д. Физика элементарных частиц и инфляционная космология. - М.: Наука, 1990. - 256с.

45 Т.е. объём пространства которых конечен.

46 Их ещё называют доменами, от англ. domain - область пространства, отмеченная некоторыми физическими особенностями.
} 
стенками. В настоящее время эта теория получила признание среди большинства специалистов ${ }^{47}$. Её философские следствия требуют глубоких и детальных исследований, здесь хотелось бы отметить, что переход к данной картине мира может являться одним из самых серьёзных в истории человечества “прорывов” в нашем видении мира. Этот переход можно назвать не только научной, но и мировоззренческой революцией. В основе её лежит переход от уни - единства к мульти - множество. Это зафиксировано и в терминологии. Всё большее количество авторов ${ }^{48}$, для обозначения подобной картины мира используют понятие Мультиверсум, вместо традиционного ещё с античности названия мира как целого - Универсума. Впрочем, философские аспекты перехода от Универсума к Мультиверсуму, есть тема отдельного, детального исследования.

\footnotetext{
${ }^{47}$ См. например: Сажин А.М. Современная космология в популярном изложении. - М.: Едиториал УРСС, $2002 .-240$ с., Рис М. Наша космическая обитель. Пер с англ. - Москва-Ижевск: Институт компьютерных исследований, 2002 192 cmp., Tegmark Max. Parallel Universes. // http://lanl.arxiv.org/abc/astro-ph/0302131 v1 и ряд других.

${ }^{48}$ См. предыдущую ссылку.
} 